\title{
Optimal Control for Combination Therapy in Cancer
}

\author{
Urszula Ledzewicz \\ Heinz Schättler \\ Alberto d'Onofrio \\ Dept. of Math. and Statistics, \\ Dept. of Electrical and Systems Engr., \\ Washington University, \\ European Institute of Oncology, \\ Southern Illinois University Edwardsville, \\ Edwardsville, Illinois, 62026-1653, \\ uledzew@siue.edu \\ St. Louis, Missouri, 63130-4899, \\ hms@wust l . edu \\ Div. of Epidemiology and Biostatistics, \\ 120141 Milan, Italy \\ alberto.donofriodieo.it
}

\begin{abstract}
A mathematical model for the scheduling of angiogenic inhibitors in combination with a killing agent is considered as an optimal control problem. Initial results on the structure of optimal controls are derived.
\end{abstract}

\section{INTRODUCTION}

A solid in vivo tumor, after going through an initial state of avascular growth, at the size of about $2 \mathrm{~mm}$ in diameter, starts the process of angiogenesis, i.e., the recruitment of surrounding host blood vessels in order to facilitate its own supply of nutrients. In absence of this process (e.g., in vitro) a tumor ceases its growth. Remarkably, through a complex bi-directional signaling mechanism, the tumor both stimulates and also inhibits the growth of endothelial cells that form the lining of the newly developing capillaries. In the early seventies J. Folkman [12] introduced the concept of anti-angiogenic therapy: a cancer treatment that targets the vasculature of a growing tumor. These treatments bring in external angiogenic inhibitors to block and in some case disrupt the growth of endothelial cells. This indirectly effects the tumor which, ideally, deprived of necessary nutrition, would regress. Contrary to traditional chemotherapy this treatment targets the genetically far more stable endothelial cells rather than the continuously mutating tumor cells. As a consequence, no clonal resistance to the angiogenic inhibitors develops in experimental cancer [2], providing a new hope for the treatment of tumors [14].

However, anti-angiogenic treatment alone only prevents the tumor from developing its blood vessel support, but does not directly destroy cancer cells. It seems clear, and this was confirmed both in vivo and in vitro, that the tumor has the tendency to grow back once treatment is halted. Numerous Phase I and II medical studies that have been and still are being conducted attest to this. More generally, due to the manifold and serious obstacles that can arise in cancer treatments, it is common not to limit treatment options to one form, but to combine different approaches in the hope of achieving synergistic effects and this also

This material is based upon research supported by the National Science Foundation under research grants DMS 0707404/0707410. U. Ledzewicz's research also was partially supported by an SIUE "Funded University Research" grant in Fall 2007. A. d'Onofrio's work was done in the framework of the European Union Integrated Project "Advancing ClinicalGenomic Trials on Cancer" (Contract no. 026996), Workpackage 2 "User needs and specifications". includes angiogenic treatments. Thus, combinations of antiangiogenesis with traditional chemotherapy are currently being pursued in clinical trials since this "simultaneously targets two compartments, the cancer cells and the vascular cells that support the tumor" (Dr. Qian, John Hopkins Kimmel Cancer Center).

Several mathematical models that describe the dynamics of angiogenesis have been proposed. Some of these aim at fully reflecting the complexity of the biological processes and allow for large-scale simulations (e.g., [1]), but then are less amenable to a mathematical analysis. But also several lowdimensional models have been formulated. Folkman and his collaborators Hahnfeldt, Panigrahy, and Hlatky, then at Harvard Medical School, developed and biologically validated a two dimensional model of ordinary differential equations for the interactions between the tumor volume, $p$, and the carrying capacity of the endothelial cells, $q$ [13]. The latter is defined as the maximum tumor volume sustainable by the vascular network. Henceforth we also refer to this as the endothelial support of the tumor for short. Based on this model, two main modifications of the original model have been formulated since then, one by Ergun, Camphausen and Wein from the National Cancer Institute in the U.S. [11], the other by d'Onofrio (at the European Institute of Oncology in Milan) and Gandolfi (at National Research Council in Rome) in [9] and [10] who also gave a mathematical analysis of the model by Hahnfeldt et al.

In [11] the important problem of how to schedule an a priori given amount of angiogenic inhibitors in such a way as to realize the maximum tumor reduction possible was proposed as an optimal control problem and initially analyzed for the problem considered there. Complete solutions to both the original model of [13] as well as its modifications from [9] and [11] were given in [16]-[18]. A different formulation of the problem was also considered in [20]. While applications of optimal control to mathematical models arising in biomedical problems have had a long history with the early focus on models in cancer chemotherapy, there has been a strong resurgence of this methodology in the analysis of newer models. This especially holds for novel treatment approaches to cancer like anti-angiogenesis discussed above or models describing the immune response to viruses (e.g., HIV [15]) or cancer and resulting immunotherapies (e.g., [5], [6], [7]), a second approach currently intensively pursued in medical 
research. In particular, for combination treatments when the overall interactions are very difficult to gauge a priori, a theoretical analysis of models can become of practical value.

All these models for anti-angiogenic therapy mentioned above are for monotherapy only and few models have been formulated for combination therapy and none so far has been analyzed mathematically. In [11] a model was presented and analyzed that combined the action of angiogenic inhibitors with radiotherapy. In the paper here, as suggested in [8], we consider the original model formulated by Hahnfeldt et al. [13] with a linear killing term added to the dynamics for the tumor growth. This is a reasonable first approximation to describe a cytotoxic killing agent in chemotherapy without considering cell-cycle specificity or it could also be considered a first crude approach to model radiotherapy ignoring the quadratic effects. Mathematically the problem now is a multi-control problem and this leads to a significantly more complex structure of possible optimal controls. In this paper we present some initial results towards a synthesis of optimal controls for these combination therapies.

\section{Mathematical Model for Anti-Angiogenic TREATMENT WITH A KILling TERM}

The underlying mathematical model was developed and biologically validated by Hahnfeldt, Panigrahy, Folkman and Hlatky [13] and has the primary tumor volume, $p$, and the carrying capacity of the vasculature, $q$, as its principal variables. A Gompertzian growth function with variable carrying capacity represented by $q$ is taken for tumor growth,

$$
\dot{p}=-\xi p \ln \left(\frac{p}{q}\right)
$$

where $\xi$ denotes a tumor growth parameter, and based on an analysis of the underlying consumption-diffusion equation in [13] the following dynamics for the endothelial support was proposed

$$
\dot{q}=b p-\left(\mu+d p^{\frac{2}{3}}\right) q-G u q
$$

This dynamics arises as a balance between stimulatory and inhibitory effects. Stimulation is modelled proportional to the tumor volume, $b p$, with $b$ a constant labelled for "birth", and inhibition consists of loss to the endothelial cells through natural causes (death etc.), $\mu q$, and inhibitory effects of the tumor given by $d p^{\frac{2}{3}} q$ with $d$ a constant labelled for "death". The power $\frac{2}{3}$ arises since inhibitors need to be released through the surface of the tumor. Generally $\mu$ is small compared to the factors and thus often is neglected $(\mu=0)$. The variable $u$ represents a control in the system and corresponds to the concentration of the inhibitors while $G$ is a constant that represents the anti-angiogenic killing parameter. In the version of the model considered here concentration and dosage of the inhibitors are identified and pharmacokinetic equations are not included.

In addition to an angiogenic inhibitor we now also consider a killing term (e.g., a cytotoxic agent in chemotherapy or a simplified model for radiotherapy) and thus modify the equation for tumor growth accordingly as

$$
\dot{p}=-\xi p \ln \left(\frac{p}{q}\right)-F p v
$$

with $v$ a second control, the concentration/dosage of a killing agent and $F$ a tumor killing parameter. We then consider the following optimal control problem:

[OC] For a free terminal time $T$, minimize the objective $J(u)=p(T)$ subject to the dynamics

$$
\begin{array}{lll}
\dot{p}=-\xi p \ln \left(\frac{p}{q}\right)-F p v, & p(0)=p_{0}, \\
\dot{q}=b p-\left(\mu+d p^{\frac{2}{3}}\right) q-G u q, & q(0)=q_{0}, \\
\dot{y}=u, & y(0)=0, \\
\dot{z}=v, & z(0)=0,
\end{array}
$$

over all piecewise continuous functions $u$ : $[0, T] \rightarrow[0, a]$ and $v:[0, T] \rightarrow[0, c]$ for which the corresponding trajectory satisfies $y(T) \leq A$ and $z(T) \leq C$.

It can be shown that for any admissible controls $u$ and $v$ and for arbitrary positive initial conditions $p_{0}$ and $q_{0}$ the corresponding solution $(p, q)$ exists for all times $t \geq 0$ and both $p$ and $q$ remain positive. Thus it is not necessary to impose this as a constraint.

The solution to problem [OC] answers the question how to schedule a priori given amounts $A$ of angiogenic inhibitors and $C$ of killing agents to achieve the maximum tumor reduction and $T$ gives the time when this tumor reduction is being achieved. From a practical point this often is the medically more relevant question to answer since resources are limited and side effects need to be kept under control. From a mathematical side, once the structure of solutions to this problem is understood, it is normally not difficult to modify the analysis to consider a fixed therapy interval $T_{t h}$ or rather than specifying the upper limit $C$ on the cytotoxic agent include this as a penalty term in the objective.

\section{NECESSARY CONDITIONS FOR OPTIMALITY}

Let $\left(u_{*}, v_{*}\right)$ be optimal controls defined over the interval $[0, T]$ with corresponding trajectory $\left(p_{*}, q_{*}, y_{*}, z_{*}\right)$. Similar to the monotherapy problem analyzed in [17], there exist degenerate initial conditions when the optimal solution is given by $T=0$. The reason for this lies in the fact that for $p<q \exp \left(-\frac{F c}{\xi}\right)$, no matter what control is being used, the cancer volume $p$ increases. Hence, if the initial condition lies in this region and if the overall amounts $A$ and $C$ are too small for the system to reach the region $p>q \exp \left(-\frac{F c}{\xi}\right)$, then the smallest value for $p$ along any solution is always given by the initial condition $p_{0}$ and mathematically the optimal solution simply is $T=0$. Other less degenerate situations are possible as well. For reasons of space, throughout this paper we simply assume that the optimal solutions have the property that $T>0$ and that all available inhibitors respectively cytotoxic agents are being used up, $y(T)=A$ and $z(T)=C$. In this case we call the 
initial condition well-posed for the optimal control problem $[\mathrm{OC}]$. It is not difficult to determine which initial conditions are well-posed (these are basic reachability questions,) but we shall not discuss this here.

For a well-posed initial condition the first-order necessary conditions for optimality of the controls $u_{*}$ and $v_{*}$ given by the Pontryagin Maximum Principle (for a recent textbook on the subject, see, e.g., [4]) state that there exist a constant $\lambda_{0} \geq 0$ and an absolutely continuous co-vector, $\lambda:[0, T] \rightarrow$ $\left(\mathbb{R}^{4}\right)^{*}$, (which we write as row-vector) such that $\left(\lambda_{0}, \lambda(t)\right) \neq$ $(0,0)$ for all $t \in[0, T]$, satisfying the adjoint equations with transversality condition,

$$
\begin{aligned}
\dot{\lambda}_{1}=\lambda_{1}(\xi & \left.\left(1+\ln \left(\frac{p_{*}(t)}{q_{*}(t)}\right)\right)+F v_{*}(t)\right) \\
& +\lambda_{2}\left(\frac{2}{3} d \frac{q_{*}(t)}{p_{*}^{\frac{1}{3}}(t)}-b\right), \quad \lambda_{1}(T)=\lambda_{0}, \\
\dot{\lambda}_{2}=-\xi \lambda_{1} \frac{p_{*}(t)}{q_{*}(t)}+\lambda_{2}\left(\mu+d p_{*}^{\frac{2}{3}}(t)+G u_{*}(t)\right), & \lambda_{2}(T)=0, \\
\dot{\lambda}_{3}=0, \quad \text { and } \quad \dot{\lambda}_{4}=0 &
\end{aligned}
$$

such that the optimal controls $u_{*}(t)$ and $v_{*}(t)$ minimize the Hamiltonian $H$,

$$
\begin{array}{r}
H=-\lambda_{1}\left(\xi p \ln \left(\frac{p}{q}\right)+F p v\right)+\lambda_{3} u+\lambda_{4} v \\
+\lambda_{2}\left(b p-\left(\mu+d p^{\frac{2}{3}}\right) q-G u q\right)
\end{array}
$$

along $\left(\lambda_{0}, \lambda(t), p_{*}(t), q_{*}(t), y_{*}(t), z_{*}(t)\right)$ over the control set $[0, a] \times[0, c]$ and the minimum value is given by 0 .

We call a pair $((p, q, y, z),(u, v))$ consisting of an admissible pair of controls $(u, v)$ and its corresponding trajectory $(p, q, y, z)$ for which there exist multipliers $\left(\lambda_{0}, \lambda\right)$ such that the conditions of the Maximum Principle are satisfied an extremal (pair) and the triple $\left((p, q, y, z),(u, v),\left(\lambda_{0}, \lambda\right)\right)$ is an extremal lift (to the cotangent bundle). Extremals with $\lambda_{0}=0$ are called abnormal while those with a positive multiplier $\lambda_{0}$ are called normal. In this case we always take $\lambda_{0}=1$.

Lemma 3.1: For well-posed initial conditions $\left(p_{0}, q_{0}\right)$ extremals are normal.

Proof. If $\lambda_{0}=0$, then by the adjoint equation both $\lambda_{1}$ and $\lambda_{2}$ vanish identically. Since the multipliers $\lambda_{3}$ and $\lambda_{4}$ are constant and the controls are non-negative, the condition that $H=\lambda_{3} u+\lambda_{4} v \equiv 0$ implies that at least one of the controls must be identically zero and thus the initial condition is illposed.

The controls are determined through the minimum condition on the Hamiltonian $H$. Since $H$ is linear in $u$ and $v$ and the control sets are compact intervals their values are determined by the values of the so-called switching functions $\Phi_{1}$ and $\Phi_{2}$ introduced above,

$$
\begin{aligned}
& \Phi_{1}(t)=\lambda_{3}-\lambda_{2}(t) G q_{*}(t), \\
& \Phi_{2}(t)=\lambda_{4}-\lambda_{1}(t) F p_{*}(t),
\end{aligned}
$$

and are given by

$$
u_{*}(t)=\left\{\begin{array}{ll}
0 & \text { if } \Phi_{1}(t)>0 \\
a & \text { if } \Phi_{1}(t)<0
\end{array} .\right.
$$

and

$$
v_{*}(t)=\left\{\begin{array}{ll}
0 & \text { if } \Phi_{2}(t)>0 \\
c & \text { if } \Phi_{2}(t)<0
\end{array} .\right.
$$

A priori the controls are not determined by the minimum condition at times when $\Phi_{i}(\tau)=0$. Clearly, if $\Phi_{i}(\tau)=0$, but $\dot{\Phi}_{i}(\tau) \neq 0$, then the control has a switch between the endpoints of the corresponding control interval at time $\tau$. On the other extreme, if $\Phi_{i}(t) \equiv 0$ on an open interval $I$, then also all derivatives of $\Phi_{i}(t)$ vanish on $I$ and this may determine the control. Controls of this kind are called singular while we refer to the constant controls as bang controls. Optimal controls then need to be synthesized from these candidates through an analysis of the switching function.

Calculations of the derivatives of the switching functions simplify significantly within the framework of geometric optimal control theory and we therefore now write the state as a 4-dimensional vector $x=(p, q, y, z)^{T}$ and express the dynamics in the form

$$
\dot{x}=f(x)+u g_{1}(x)+v g_{2}(x)
$$

where

$$
f(x)=\left(\begin{array}{c}
-\xi p \ln \left(\frac{p}{q}\right) \\
b p-\left(\mu+d p^{\frac{2}{3}}\right) q \\
0 \\
0
\end{array}\right)
$$

$$
g_{1}(x)=\left(\begin{array}{c}
0 \\
-G q \\
1 \\
0
\end{array}\right) \text {, and } g_{2}(x)=\left(\begin{array}{c}
-F p \\
0 \\
0 \\
1
\end{array}\right) \text {. }
$$

Following differential geometric conventions we denote the canonical basis vectors by $\frac{\partial}{\partial p}=(1,0,0,0)^{T}, \frac{\partial}{\partial q}=$ $(0,1,0,0)^{T}$ etc. This allows to write these vector fields and their brackets in a space saving form, e.g.,

$$
g_{1}(x)=-G q \frac{\partial}{\partial q}+\frac{\partial}{\partial y}, \quad g_{2}(x)=-F p \frac{\partial}{\partial p}+\frac{\partial}{\partial z} .
$$

The derivatives of the switching function can easily be computed using the following well-known result that can be verified by a direct calculation.

Proposition 3.1: Let $h$ be a continuously differentiable vector field and define $\Psi(t)=\langle\lambda(t), h(x(t))\rangle$. Then the derivative of $\Psi$ along a solution to the system equation (16) for control $u$ and a solution $\lambda$ to the corresponding adjoint equations (8)-(10) is given by

$$
\dot{\Psi}(t)=\left\langle\lambda(t),\left[f+u g_{1}+v g_{2}, h\right](x(t))\right\rangle,
$$

where $[f, h]$ denotes the Lie bracket of the vector fields $f$ and $h$. Recall that the Lie bracket is computed in local coordinates as $[f, h](x)=D h(x) f(x)-D f(x) h(x)$ with $D f$ denoting the matrix of the partial derivatives of $f$. 


\section{Analysis of Singular Controls}

In this notation the switching functions are given as

$$
\Phi_{1}(t)=\left\langle\lambda(t), g_{1}(x(t))\right\rangle, \quad \Phi_{2}(t)=\left\langle\lambda(t), g_{2}(x(t))\right\rangle,
$$

and hence

$$
\begin{aligned}
& \dot{\Phi}_{1}(t)=\left\langle\lambda(t),\left[f+v g_{2}, g_{1}\right](x(t))\right\rangle, \\
& \dot{\Phi}_{2}(t)=\left\langle\lambda(t),\left[f+u g_{1}, g_{2}\right](x(t))\right\rangle .
\end{aligned}
$$

An elementary calculation verifies that $g_{1}$ and $g_{2}$ commute, $\left[g_{1}, g_{2}\right] \equiv 0$, and thus this simplifies to

$$
\begin{aligned}
& \dot{\Phi}_{1}(t)=\left\langle\lambda(t),\left[f, g_{1}\right](z(t))\right\rangle, \\
& \dot{\Phi}_{2}(t)=\left\langle\lambda(t),\left[f, g_{2}\right](z(t))\right\rangle .
\end{aligned}
$$

As a consequence we have that

Lemma 4.1: The controls $u$ and $v$ cannot both be singular on an open interval $I$ simultaneously .

Proof. Direct computations verify that

$$
\left[f, g_{1}\right](x)=G p\left(\xi \frac{\partial}{\partial p}-b \frac{\partial}{\partial q}\right)
$$

and

$$
\begin{aligned}
{\left[f, g_{2}\right](x) } & =F p\left(-\xi \frac{\partial}{\partial p}+\left(b-\frac{2}{3} d p^{-\frac{1}{3}} q\right) \frac{\partial}{\partial q}\right) \\
& =-\frac{F}{G}\left[f, g_{1}\right](x)-\frac{2}{3} F d p^{\frac{2}{3}} q \frac{\partial}{\partial q} .
\end{aligned}
$$

It follows that the four vector fields $g_{1}, g_{2},\left[f, g_{1}\right]$, and $\left[f, g_{2}\right]$ are linearly independent everywhere. But the multiplier $\lambda$ is non-zero since $\lambda_{1}$ and $\lambda_{2}$ satisfy a homogeneous linear ODE with non-zero terminal condition and thus $\lambda$ cannot vanish against all four vector fields at any time.

It is not difficult to see that this implies that if one of the controls is singular on an interval $I$, then the other control has isolated switchings on $I$. Because of space restrictions here we only consider the more relevant case of $u$-singular controls and assume that $\Phi_{1}(t) \equiv 0$ on an open interval $I=(\alpha, \beta)$ and that $v$ is constant. Hence $\dot{\Phi}_{1}(t)=\left\langle\lambda(t),\left[f, g_{1}\right](x(t))\right\rangle \equiv 0$ and

$$
\ddot{\Phi}_{1}(t)=\left\langle\lambda(t),\left[f+u g_{1}+v g_{2},\left[f, g_{1}\right]\right](x(t))\right\rangle \equiv 0 \text {. }
$$

Except for the extra fourth coordinate which is 0 the vector fields $f$ and $g_{1}$ are the same ones as for the case of monotherapy considered in [17] and a direct calculation verifies that

$$
\left[g_{1},\left[f, g_{1}\right]\right](x(t))=-G^{2} b p \frac{\partial}{\partial q} .
$$

so that

$$
\left\langle\lambda(t),\left[g_{1},\left[f, g_{1}\right]\right](x(t))\right\rangle=-\lambda_{2}(t) G^{2} b p .
$$

Along an optimal $u$-singular control the multipliers $\lambda_{2}$ and $\lambda_{3}$ must be positive: For, $\lambda_{3}$ is constant and the fact that the switching function $\Phi_{1}(t)=\lambda_{3}-\lambda_{2}(t) G q(t)$ vanishes on $I$ implies that $\lambda_{2}$ and $\lambda_{3}$ have the same sign on $I$. But if $\lambda_{3}<$ 0 , then the Legendre-Clebsch condition for minimality of the singular control (with $v$ constant) is violated (e.g., [3]) and the degenerate case $\lambda_{3}=0$ is not possible since this would imply $\lambda_{2}(t) \equiv 0$ on $I$ and then by the adjoint equation also $\lambda_{1}(t) \equiv 0$ on $I$ which is not possible. Thus along an optimal $u$-singular control we have that $\left\langle\lambda(t),\left[g_{1},\left[f, g_{1}\right]\right](x(t))\right\rangle<0$ and the singular control can be expressed as

$$
u_{*}(t)=-\frac{\left\langle\lambda(t),\left[f+v g_{2},\left[f, g_{1}\right]\right](x(t))\right\rangle}{\left\langle\lambda(t),\left[g_{1},\left[f, g_{1}\right]\right](x(t))\right\rangle} .
$$

Another computation shows that

$$
\left[g_{2},\left[f, g_{1}\right]\right](x(t))=F G b p \frac{\partial}{\partial q}=-\frac{F}{G}\left[g_{1},\left[f, g_{1}\right]\right](x(t))
$$

and we can therefore write

$$
u_{*}(t)=-\frac{\left\langle\lambda(t),\left[f,\left[f, g_{1}\right]\right](x(t))\right\rangle}{\left\langle\lambda(t),\left[g_{1},\left[f, g_{1}\right]\right](x(t))\right\rangle}+\frac{F}{G} v .
$$

The first term in this expression is exactly the singular control for the monotherapy case computed in [17] and we can simply draw on these results to determine the singular controls. It follows from those calculations that $\left[f,\left[f, g_{1}\right]\right]$ lies in the linear span of the vector fields $\left[f, g_{1}\right]$ and $\left[g_{1},\left[f, g_{1}\right]\right]$,

$$
\left[f,\left[f, g_{1}\right]\right](x)=\left(\xi+b \frac{p}{q}\right)\left[f, g_{1}\right](x)-\psi\left[g_{1},\left[f, g_{1}\right]\right](x)
$$

with

$$
\psi(p, q)=\frac{1}{G}\left(\xi \ln \left(\frac{p}{q}\right)+b \frac{p}{q}+\frac{2}{3} \xi \frac{d}{b} \frac{q}{p^{\frac{1}{3}}}-\left(\mu+d p^{\frac{2}{3}}\right)\right) .
$$

Overall we therefore have shown the following result:

Proposition 4.1: If the optimal control $u_{*}$ is singular on an open interval $I$ and $v_{*}$ is constant, then

$$
u_{*}(t)=\psi\left(p_{*}(t), q_{*}(t)\right)+\frac{F}{G} v_{*} .
$$

Note that the necessary condition $H \equiv 0$ of the Maximum Principle implies in addition that

$$
\left\langle\lambda(t), f(x(t))+v g_{2}(x(t))\right\rangle \equiv 0
$$

and thus the multiplier $\lambda(t)$ must vanish against the three vector fields $f+v g_{2}, g_{1}$ and $\left[f, g_{1}\right]$. This now leads to qualitatively very different structures depending on whether $v=0$ or $v=c>0$.

Case 1: If $v \equiv 0$ on $I$, then naturally the problem reduces to the monotherapy situation analyzed in [17]. In this case the vector fields $f, g_{1}$ and $\left[f, g_{1}\right]$ all have zero last coordinate and span the $(p, q, y)$-subspace. The only vectors orthogonal to all of these are $\left(0,0,0, \lambda_{4}\right)$. But along a singular arc the component $\lambda_{2}$ must be positive and thus it follows that the vector fields $f, g_{1}$ and $\left[f, g_{1}\right]$ must be linearly dependent along the singular arc. Hence, although formulated in $\mathbb{R}^{4}$, this case reduces to the three-dimensional problem considered in [17]. Under the natural condition that

$$
G a>b-\mu>0,
$$

that is, in principle the maximum dosage of outside inhibitors is able to overcome the net effect of stimulation through 
the tumor minus natural death terms (the coefficient $b$ is generally much larger then $\mu$ and the second inequality is not a realistic restriction) we thus have the following result:

Proposition 4.2: [17] If an optimal control $u_{*}$ is singular on an interval $(\alpha, \beta)$ and $v \equiv 0$ on $(\alpha, \beta)$, then the corresponding trajectory $\left(p_{*}, q_{*}\right)$ lies on a uniquely singular curve $\mathcal{S}$. Defining new variables $(p, r)$ with $r=\frac{p}{q}$, this curve $\mathcal{S}$ can be parameterized in the form

$$
p^{2}+\left(\frac{b}{d} r(\ln r-1)+\frac{\mu}{d}\right)^{3}=0
$$

with $r$ in some interval $\left[r_{1}, r_{2}\right] \subset(0, \infty)$. The singular control keeps the system on the singular curve and is given as a feedback function of $r$ in the form

$$
u_{\sin }(r)=\frac{1}{G}\left[\left(\frac{1}{3} \xi+b r\right) \ln r+\frac{2}{3} \xi\left(1-\frac{\mu}{b r}\right)\right] .
$$

There exists exactly one connected arc on the singular curve $\mathcal{S}$ along which the singular control is admissible, i.e., satisfies the bounds $0 \leq u_{\sin }(r) \leq a$. This arc is defined over an interval $\left[r_{\ell}^{*}, r_{u}^{*}\right]$ where $r_{\ell}^{*}$ and $r_{u}^{*}$ are the unique solutions to the equations $u_{\sin }\left(r_{\ell}^{*}\right)=0$ and $u_{\sin }\left(r_{u}^{*}\right)=a$. At these points the singular control saturates at the control limits $u=0$ and $u=a$.

Figs. 1 and 2 illustrate the proposition for the following parameter values taken from [13] that were obtained by fitting experimental data for the case of Lewis lung carcinomas implanted in mice: The variables $p$ and $q$ are volumes measured in $\mathrm{mm}^{3} ; \xi=\frac{0.192}{\ln 10}=0.084$ per day (adjusted to the natural logarithm), $b=5.85$ per day, $d=0.00873$ per $\mathrm{mm}^{2}$ per day, $G=0.15 \mathrm{~kg}$ per $\mathrm{mg}$ of dose per day with concentration in $\mathrm{mg}$ of dose per $\mathrm{kg}$, and for illustrative purposes we chose a small positive value for $\mu, \mu=0.02$ per day. Since we identify dosage and concentration, both $a$ and $A$ are in units of concentration and just for illustrative purposes we picked $a=75$ and $A=300$. Fig. 1 shows the plot for the singular control defined by (31) also indicating the values $r_{\ell}^{*}$ and $r_{u}^{*}$ where the control saturates at $u_{\sin }(r)=0$ and $u_{\sin }(r)=a$. Fig. 2 shows the graph of the singular curve given by formula (30). Saturation restricts the admissible part to the curve that is marked with a solid line in Fig. 2. The qualitative structures shown in these figures are generally valid for arbitrary parameter values both for the control and the singular curve. With decreasing values for the upper control limit $a$ the admissible portion shrinks, but it is always preserved.

Case 2: If $v \equiv c>0$ on $I$, there exists an up to multiples unique multiplier $\lambda$ that is orthogonal to the vector fields $f+c g_{2}, g_{1}$ and $\left[f, g_{1}\right]$. Thus now there are no restraints on the locus of where the singular control is admissible and the singular control is a feedback in $(p, q)$-space. Fig. 3 below shows the admissible portion of this feedback control for the parameter values given earlier.

\section{Comparison of Singular Protocols with ReAlizable BANG-BANG Strategies}

It then becomes necessary to synthesize optimal controls from all possible candidates including partially singular

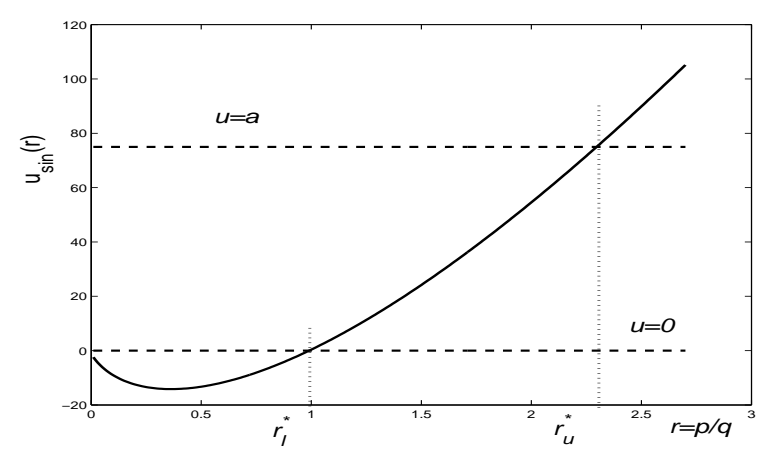

Fig. 1. The singular control $u_{\text {sin }}(r)$ while $v=0$

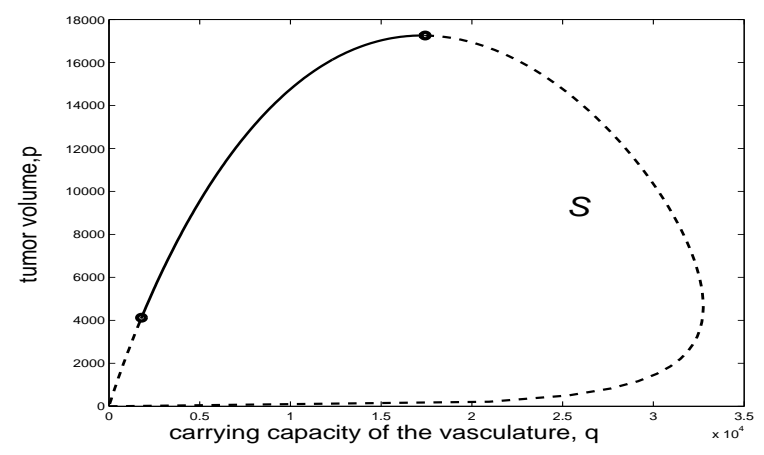

Fig. 2. The admissible singular curve $\mathcal{S}$ for $v=0$

pieces. For the monotherapy analyzed in [17] the singular arc was the center piece anchoring this synthesis. A typical optimal control followed the control $u=a$ (for initial conditions with a low $q_{0}$-value also $u=0$ is possible) until it reached the singular arc and then followed the singular arc until all inhibitors had been exhausted. Here, with the complexity of the 2-control system the structure becomes more complicated and the full synthesis is still under investigation. Below we include two graphs that compare two types of candidates for optimal controls for different initial conditions. The blue trajectories correspond to simply using both controls from the beginning at full doses until they run out (for the monotherapy this is a rather good sub-optimal strategy); the red trajectory corresponds to giving full dose of the control $v$, the cytotoxic agent, until the drugs are exhausted while the angiogenic inhibitors, the control $u$, at the beginning follows the singular regimen computed above and then when the cytotoxic agents are exhausted follows the optimal control for the monotherapy problem. In both cases the tumor volume is at $12,000 \mathrm{~mm}^{3}$ initially and the endothelial support initially is at $6,000 \mathrm{~mm}^{3}$ in Fig. 4 and at $12,000 \mathrm{~mm}^{3}$ in Fig. 5. It is seen from the graphs that the full dose strategy does better for the higher $q_{0}$-value while the singular regimen is the better strategy for the smaller endothelial support.

\section{CONCLUSION}

We presented some preliminary results about optimal controls for a mathematical model that combines anti-angiogenic 


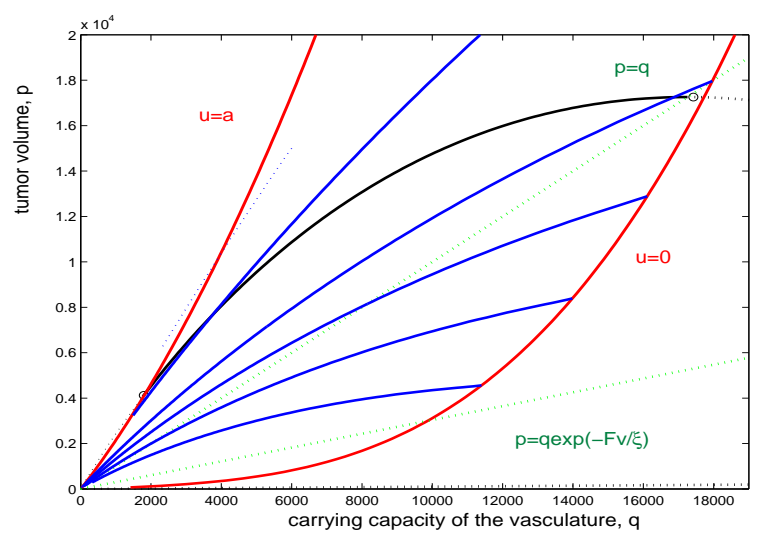

Fig. 3. The admissible portion of the singular flow in $u$ for $v=c$

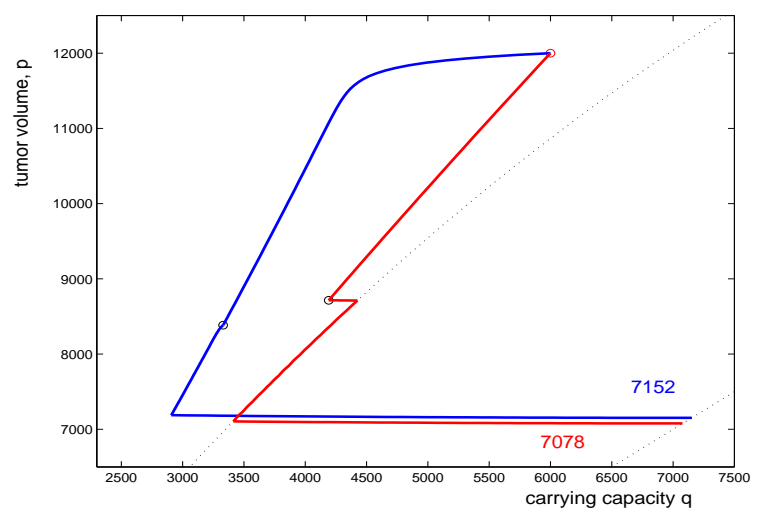

Fig. 4. A comparison of full-dose with singular protocols for $p_{0}=12,000$ and $q_{0}=6,000$

therapy with a chemotherapeutic killing agent. Mathematically this becomes a multi-control problem and the structure of a synthesis of optimal controls is significantly more complex than in the monotherapy case. Singular controls, especially $u$-singular controls for the angiogenic inhibitors, will again be part of this synthesis, but its full structure in the sense of a regular synthesis [19] still needs to be worked out. From this point of view, also the mathematically simpler, but biologically intriguing problem of chemotherapeutic agents that have an anti-angiogenic effect is of interest. In this case the mathematical problem again is single-input with $u(t)$ an increasing function of $v(t)$.

\section{REFERENCES}

[1] A. Anderson and M. Chaplain, Continuous and discrete mathematical models of tumor-induced angiogenesis, Bull. Math. Biol., 60, 1998, pp. $857 \mathrm{ff}$

[2] T. Boehm, J. Folkman, T. Browder, and M.S. O’Reilly, Antiangiogenic therapy of experimental cancer does not induce acquired drug resistance, Nature, 390, (1997), pp. 404ff

[3] B. Bonnard and M. Chyba, Singular Trajectories and their role in Control Theory, Math'ematiques \& Applications, vol. 40, Springer Verlag, Paris, 2003

[4] A. Bressan and B. Piccoli, Introduction to the Mathematical Theory of Control, American Institute of Mathematical Sciences, 2007

[5] F. Castiglione and B. Piccoli, Optimal control in a model of dendritic cell transfection cancer immunotherapy, Bulletin of Mathematical Biology, 68, (2006), pp. 255-274

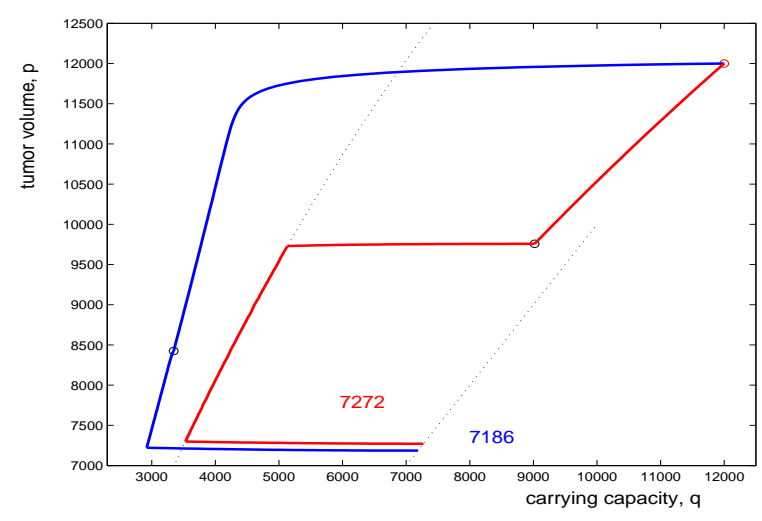

Fig. 5. A comparison of full-dose with singular protocols for $p_{0}=12,000$ and $q_{0}=12,000$

[6] L.G. de Pillis and A. Radunskaya, A mathematical tumor model with immune resistance and drug therapy: an optimal control approach, $J$. of Theoretical Medicine, 3, (2001), pp. 79-100

[7] A. d'Onofrio, A general framework for modeling tumor-immune system competition and immunotherapy: mathematical analysis and biomedical inferences, Physica D, 208, 2005, pp. 220-235

[8] A. d'Onofrio, Rapidly acting antitumoral anti-angiogenic therapies, Physical Review E, 76 (3), Art. No. 031920, 2007

[9] A. d'Onofrio and A. Gandolfi, Tumour eradication by antiangiogenic therapy: analysis and extensions of the model by Hahnfeldt et al., Math. Biosci., 191, 2004, pp. 159-184

[10] A. d'Onofrio and A. Gandolfi, The response to antiangiogenic anticancer drugs that inhibit endothelial cell proliferation, Appl. Math. and Comp., 181, 2006, pp. 1155-1162

[11] A. Ergun, K. Camphausen and L.M. Wein, Optimal scheduling of radiotherapy and angiogenic inhibitors, Bull. of Math. Biology, 65 , (2003), pp. 407-424

[12] J. Folkman, Antiangiogenesis: new concept for therapy of solid tumors, Ann. Surg., 175, (1972), pp. 409-416

[13] P. Hahnfeldt, D. Panigrahy, J. Folkman and L. Hlatky, Tumor development under angiogenic signaling: a dynamical theory of tumor growth, treatment response, and postvascular dormancy, Cancer Research, 59, (1999), pp. 4770-4775

[14] R.S. Kerbel, A cancer therapy resistant to resistance, Nature, 390 , (1997), pp. 335-336

[15] D. Kirschner, S. Lenhart, and S. Serbin, Optimal control of chemotherapy of HIV, J. Math. Biol., 35, (1997), pp. 775-792

[16] U. Ledzewicz and H. Schättler, A synthesis of optimal controls for a model of tumor growth under angiogenic inhibitors, Proc. 44th IEEE Conf. on Dec. and Contr., Sevilla, Spain, 2005, pp. 945-950

[17] U. Ledzewicz and H. Schättler, Anti-Angiogenic therapy in cancer treatment as an optimal control problem, SIAM J. Contr. Optim., $\mathbf{4 6}$ 2007, pp. 1052-1079

[18] U. Ledzewicz and H. Schättler, Analysis of a mathematical model for tumor anti-angiogenesis, Opt. Contr. Appl. Meth., 29, (1), (2008), pp. 41-57

[19] B. Piccoli and H.J. Sussmann, Regular synthesis and sufficiency conditions for optimality, SIAM J. Control Optimization, 39, (2000), pp. $359-410$

[20] A. Swierniak, A. d'Onofrio and A. Gandolfi, Control Problems related to tumor angiogenesis, Proceedings of IECON 2006, IEEE Press (2006), pp. 677-681 